\title{
BCINet: An Optimized Convolutional Neural Network for EEG-Based Brain-Computer Interface Applications
}

\author{
Avinash Kumar Singh \\ Australian Artificial Intelligence Institute \\ University of Technology Sydney \\ Ultimo, Australia \\ avinash.singh@uts.edu.au
}

\author{
Xian Tao \\ Research Center of Precision Sensing and Control \\ Institute of Automation \\ Chinese Academy of Sciences \\ Beijing, China \\ taoxian2013@ia.ac.cn
}

\begin{abstract}
EEG based brain-computer interface (BCI) allows people to communicate and control external devices using brain signals. The application of $\mathrm{BCI}$ ranges from assisting in disabilities to interaction in a virtual reality environment by detecting user intent from EEG signals. The major problem lies in correctly classifying the EEG signals to issue a command with minimal requirement of pre-processing and resources. To overcome these problems, we have proposed, BCINet, a novel optimized convolution neural network model. We have evaluated the BCINet over two EEG based BCI datasets collected in mobile brain/body imaging (MoBI) settings. BCINet significantly outperforms the classification for two datasets with up to $20 \%$ increase in accuracy while fewer than $75 \%$ trainable parameters. Such a model with improved performance while less requirement of computation resources opens the possibilities for the development of several real-world BCI applications with high performance.

Index Terms-Convolutional neural network, deep learning, EEG, brain-computer interface, MOBI, cognitive conflict, BCINet.
\end{abstract}

\section{INTRODUCTION}

Brain-Computer Interface (BCI) allows people to communicate and control external devices using brain signals. Originally BCI was envisioned to help individuals with disabilities such as amyotrophic lateral sclerosis, cerebral palsy, stroke, or spinal cord injury. BCI has proven to be significantly useful in rehabilitation and social cognition after strokes such as Attention deficit hyperactivity disorder (ADHD) and Autism [1] In recent years, more application has been proposed not limited to the user with a disability but also health users, e.g., BCI based drone flying, in gaming like car racing, three color matching, interaction in virtual reality, etc. A BCI recognizes the user's intent through the electrophysiological signals, which are usually recorded over the scalp, underneath the scalp, or within the brain. Other types of physiological signals can be recorded by magnetic sensors, infrared sensors, or by other means. One such method of recording physiological signals is the electroencephalogram (EEG). EEG records the change in the postsynaptic potential of cortical neurons across the scalp using electrodes [2]. EEG is widely famous in $\mathrm{BCI}$ research, and its applications, because of its noninvasive method with high temporal resolution. EEG has also preferred choice because of its portability and affordability, but one the other hand, it is highly susceptible to electrical noise and has low spatial resolution [3]. The use of EEG signals to develop $\mathrm{BCI}$ poses several challenges which usually overcome with the help of machine learning.

The past few years have seen an increased number of deep learning applications in understanding and classifying brain signals [4]. Deep learning is already shown a high number of successful applications in the field of natural language processing [5] and computer vision [6]. Deep learning has a property to learn valuable information from raw data without manual labour [7], which is very useful in EEG signal processing for BCI. Convolutional neural network $(\mathrm{CNN})$ is one of the prevalent methods in the field of deep learning. It has proven to effective model in several applications of BCI, such as epilepsy/seizures prediction [8], [9], for detection of visualevoked responses [10], motor imagery classification [11], and speller [12]. Although deep learning can learn from raw EEG data, it required pre-processing signals to reach the optimal performance level. The processing method is highly dependent on individual data sets and domain knowledge, which could vary task by task. On the other hand, it is also possible that the pre-processed data does not contain all the information and potentially could be excluded the relevant EEG features depend on the choice of pre-processing such as different filtering [13], channel referencing [14], etc. The use of methods that can learn from raw EEG data without the hand-crafted method for pre-processing is highly desirable, particularly for BCI applications.

However, deep learning methods have shown promising results to automate the process to learn from raw EEG data [10][12] with high performance but come with a considerable resources cost such as computation and space. Such a cost poses a hurdle to develop real-world BCI applications with limited resources. It is also crucial to develop a method that can show high performance by utilizing deep learning to get richer representation while minimizing resources re- 
quirements. There could be two significant ways to reduce the resource cost with deep learning methods. By using the fixed weight matrices with deep learning methods like CNN using approximations such as SVD [15]. Another possible way, which this paper is focused on, by reducing the complexity of deep learning network itself while preserving the high performance. There have been several works proposed particularly in the past [16]-[18] to reduce the complexity of model but no work found focused on the model developed for EEG-based BCI applications.

In the line of this work, we have introduced BCINet for BCI applications with the property of significantly reduced complexity of model with better performance. The proposed model has been evaluated with two noisy EEG datasets collected in MoBI [19] settings of different sizes. Our results from the BCINet show a significant reduction in trainable parameters such that an optimized model and significantly improved performance to classify EEG signals compared to some of the popular deep learning model [20]-[22]. The significant contributions of presented work are as follows:

1) A novel optimized deep learning model, BCINet is proposed for EEG based BCI applications.

2) The significantly less trainable parameters compare to existing models for EEG based BCI applications.

3) The significantly improved performance compare to existing models for EEG based BCI applications.

\section{MATERIALS AND METHOD}

\section{A. Data description}

The proposed method has been tested on two datasets collected in MoBI settings in cognitive conflict task. The description of datasets is below:

1) CC-dataset: Cognitive Conflict in the $3 D$ object selection task: The 62-channel EEG dataset (sampling rate $1000 \mathrm{~Hz}$ ) is collected with 16 participants while performing $3 \mathrm{D}$ object selection tasks with their dominant hand tracked by the Leap Motion controller in virtual reality (VR). Fig. 11 top) displays the scenario where the user is performing the task wearing an EEG cap together with a VR headset, while Fig. 1(bottom) displays the whole task in a trial. The trial starts with a cube appearing on the table, and the participants were instructed to reach out and select (touch) the cube in VR. The cube would turn red when it was touched by the participant and identified as a normal condition in EEG signals. In other cases, the cube would turn red prematurely and identified as a cognitive conflict condition in EEG signals. For more detail about the experiment and data, please see [23], [24].

For the proposed CNN model, the trial has been extracted from EEG signals $200 \mathrm{~ms}$ prior to the onset of cube touch to $1000 \mathrm{~ms}$ after it, and about 500 trials have been extracted from each participant data. Overall, the dimension of the total data was $62 \times 1200 \times 6841$, where 5,075 trials belonged to non-conflict class and 1,766 for conflict class.

2) pHRC-dataset: Cognitive Conflict in physical humanrobot collaboration ( $p H R C$ ): The 32-channel EEG dataset (sampling rate $1000 \mathrm{~Hz}$ ) is collected with 10 participants while

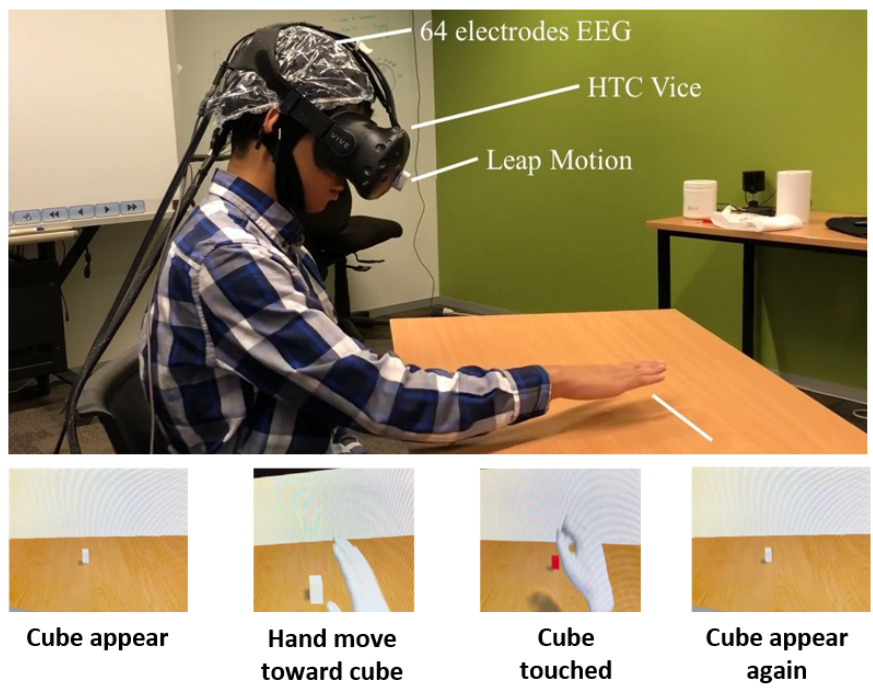

Fig. 1. An experiment scenario in CC-dataset where the participant is trying to touch a cube in VR environment. (see more detail in [23], [24])

performing a swing game task using pHRC. Fig. 2(top) displays the participant is performing the task holding a robotic arm in the hand and Fig. 2(bottom) displays the structure of the task. In the task, the participant needs to move the robotic arm from the center to left or right randomly as instructed on the project screen represented by a green target. Once the user reaches the target, the target would turn red as a sign to finish. The blue circle represents the point the nozzle of the robot is aiming at. In order to keep the user more engaged additional input from the user was asked to perform like holding the back handle known as the robot end-effector. All the trials where participants successfully move and reach the target identified as normal in EEG signals while in other cases, the task was suddenly stopped by a virtual object and identified as a conflict in EEG signals. For more detail about the whole experiment, please check [25].

For the proposed CNN model, the 250 trials have been extracted from $200 \mathrm{~ms}$ prior to the onset of cube touch to $1000 \mathrm{~ms}$ after it. The overall dimension of the data for a participant was $32 \times 1200 \times 5600$, where 3,354 trials belonged to non-conflict class and 2,246 for conflict class.

$\mathrm{CC}$ and pHRC-dataset have been divided into $60 \%, 20 \%$, $20 \%$ for training, validation, and testing respectively for binary conditions using stratified sampling method [26] to avoid the imbalanced class problem. See Table I for data description summary.

TABLE I

DESCRIPTION OF CC AND PHRC-DATASET USED

$\begin{array}{ccccc}\text { Datasets } & \text { Channels } & \text { Sampling Rate } & \text { Classes } & \text { Dimension } \\ \text { CC } & 64 & 1000 & 2 & 62 \times 1200 \times 6841 \\ \text { pHRC } & 32 & 1000 & 2 & 32 \times 1200 \times 5600\end{array}$



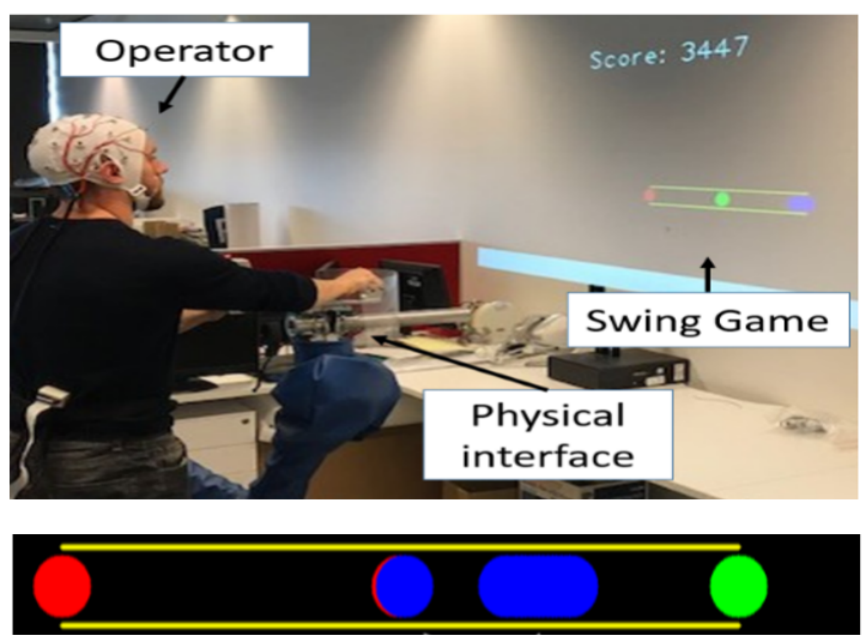

Non-target

Starting point and area covered by moving pHRC

Target location to move pHRC

Fig. 2. An experiment scenario in pHRC-dataset where participant is using a robot to perform a task. (see more detail in $[25 \mid$ )

\section{B. Proposed model-BCINet}

As shown in Fig. 3, the BCINet contains two parallel layers of the $2 \mathrm{D}$ convolution layer for the spatial conversion of data with a filter of size 64 and 32. The output of both 2D convolution layers has been concatenated. The output of 2D convolution layers also uses to create a squeeze and excitation layer with the help of global average pooling, followed by a full-connected layer. The concatenated layer and excited layer have been multiplied to get a richer representation of features. The resultant input is fed into one sequential and two parallel 2D depthwise separable convolution layers with different filter $=32,32$, and 64 with kernel $=3,64,32$. The output of the $2 \mathrm{D}$ depthwise separable convolution has been flattened to receive reshaped tensor for classification. The resultant output use 'softmax' for classification. The activation layer utilizes the exponential linear unit (elu) with a linear dropout layer at each layer of 0.5 for classification.

The BCINet is fitted using Adam optimizer, using default parameters defined in [27]. We ran 300 training epochs and performed validation on each epoch, saving the model weights, which lowest validation set loss. All models were trained on NVIDIA Quadro P5000 GPU, with CUDA 9 and cuDNN v7, in TensorFlow [28], using the Keras API [29].

\section{Baseline model description}

EEGNet. The EEGNet [20] is CNN based model that contains 2D convolution layer, 2D depthwise convolution layer, and 2D separable convolution layer followed by 'softmax' for classification with 'adam' as an optimizer. As per the model's recommendation, we have used a batch size of 16 , kernel length of 500, and a 0.5 dropout rate. Other parameters used in EEGNet included $\mathrm{F} 1=$ number of EEG channels, $\mathrm{D}=2$, and $\mathrm{F} 2=\mathrm{F} 1 * \mathrm{D}$. With $\mathrm{F} 1$ being the number of temporal filters, $\mathrm{D}$ the number of spatial and $\mathrm{F} 2$ the number of pointwise filters.
DeepConvNet. It consists of four convolutional blocks and a classification block. The first convolutional block is to handle EEG inputs, followed by three standard convolution layers. The classification is performed using a softmax with Adam optimizer. We have used batch size $=16$, dropout rate $=$ 0.50 with 300 epochs. The number of filters used in four convolution layers was $25,50,100$, and 200 , respectively, with each layer consist of five kernels [21].

ShallowNet. It is a shallow version of DeepConvNet, inspired by filter bank common spatial patterns. It consists of two convolution layers, followed by a fully connected layer. The classification is performed using a softmax with Adam optimizer. We have used batch size $=16$, dropout rate $=0.50$ with 300 epochs. The number of filters used in two convolution layers was 40, respectively, with each layer consist of kernel size $=13$ [22].

\section{Evaluation metrics}

The parameters for all the classifiers compared in this paper have been set up before training and testing for all participants. In this work, the classes are imbalanced; therefore, stratified random sampling [26] has been used on the data with the aforesaid machine learning algorithms. To compare the results of different classifiers, we have evaluated overall accuracy, precision, recall, and F1-score for a targeted class.

\section{RESUlTS AND DISCUSSION}

\section{A. Comparison between parameters}

We have compared the parameters required by the BCINet model with EEGNet, ShallowNet, and DeepConvNet. As shown in Table III It can be seen that BCINet needed about $72 \%$ less trainable parameters compare to EEGNet, $75 \%$ less trainable compare to DeepConvNet, and 55\% less trainable parameters compare to ShallowNet for CC-dataset. Similarly, BCINet required about $70 \%$ less trainable parameters compared to DeepConvNet, $17 \%$ less trainable than ShallowNet, and $8 \%$ less trainable parameters compare to EEGNet for pHRC-dataset.

In summary, BCINet required up to $75 \%$ fewer parameters for CC-dataset and about $70 \%$ fewer parameters for pHRCdataset than baseline models. Less requirement of parameter also implies less necessity of computational resources such as time and complexity. It is also known that fewer parameter requirements also suggest less need for data to learn new features in deep learning.

On the other hand, the BCINet has $69 \%$ less prediction time compare to EEGNet while no improvement compared to ShallowNet and DeepConvNet for CC-dataset. Similarly, BCINet has $50 \%$ less prediction time compared to EEGNet, while again, there is no improvement compared to ShallowNet and DeepConvNet for pHRC-dataset. The major reason behind no improvement in prediction time in BCINet compare to ShallowNet and DeepConvNet is the use of a depthwise separable convolution layer that generally requires significantly higher computation time compared to normal convolution layer [30]. The better prediction time could speed up the whole system's 


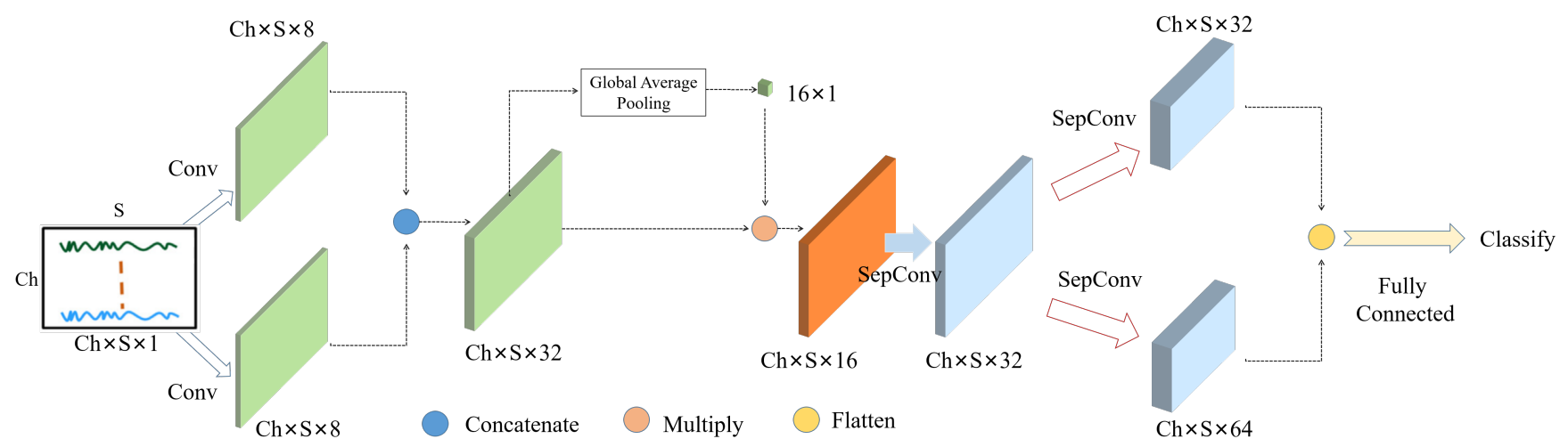

Fig. 3. Architecture of BCINet

TABLE II

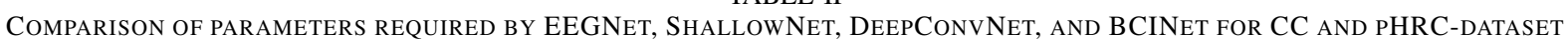

\begin{tabular}{ccccccccc} 
& \multicolumn{2}{c}{ EEGNet } & \multicolumn{2}{c}{ ShallowNet } & \multicolumn{2}{c}{ DeepConvNet } & \multicolumn{2}{c}{ BCINet } \\
Params & CC & pHRC & CC & pHRC & CC & pHRC & CC & pHRC \\
Trainable & 180,794 & 58,754 & 112,642 & 64,642 & 199,527 & 180,777 & 50,674 & 53,704 \\
Non-Trainable & 63,736 & 32,896 & 600 & 600 & 875 & 875 & 8,088 & 8,238 \\
Total & 244,530 & 91,650 & 113,242 & 65,242 & 200,402 & 181,652 & 58,762 & 61,942
\end{tabular}

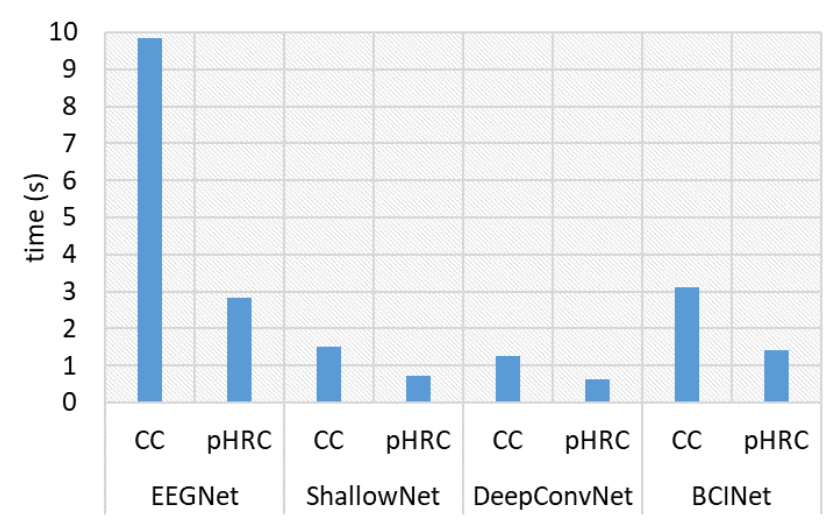

Fig. 4. Time required for prediction in EEGNet, ShallowNet, DeepConvNet, and BCINet on CC and pHRC-dataset

performance, which is very improvement for BCI applications with higher information transfer rate (ITR) [31].(Also see Fig. 4).

\section{B. Classification performance}

We have also evaluated the classification efficacy of the BCINet with EEGNet, ShallowNet, and DeepConvNet. It can be seen from Fig. 5 and Table III] that, BCINet significantly outperformed compare to baseline models. BCINet shows an accuracy improvement by $10 \%$ for EEGNet, $5 \%$ for DeepConvnet, and a slight increase in ShallowConvNet for CCdataset. Similarly, BCINet shows an accuracy improvement by $20 \%$ for EEGNet, $31 \%$ for ShallowNet, and 9\% for DeepConvnet pHRC-dataset. This is a significant improvement in classification compare to the baseline model while using significantly fewer parameters.
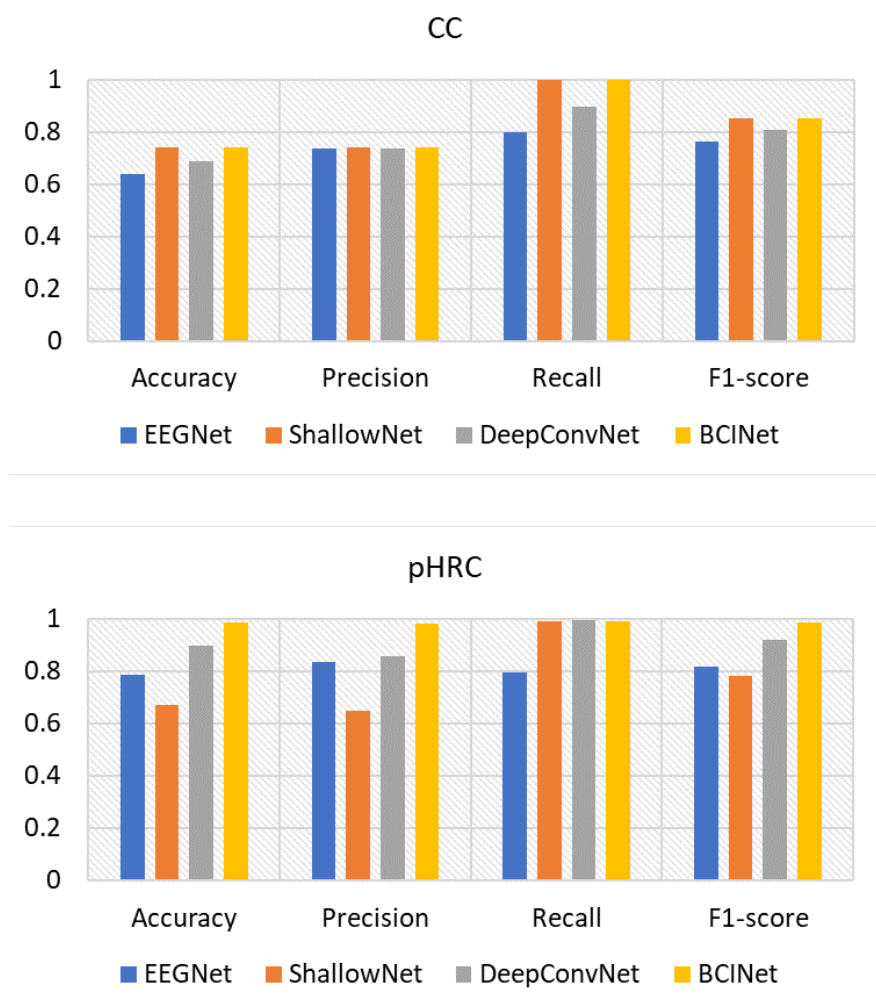

Fig. 5. Accuracy, precision, recall, and F1-score for EEGNet, ShallowNet, DeepConvNet, and BCINet on CC and pHRC-dataset 
As mentioned earlier, $\mathrm{CC}$ and pHRC-datasets has an imbalanced class problem. Therefore, we also look at other performance indicators such as precision, recall, and F1-score for BCINet and all baseline models. Again, as shown in Fig. 5. BCINet outperformed compare to all baseline models in these indicators. However, interestingly, BCINet only slightly improved compared to ShallowNet for CC-dataset similar to accuracy. One potential reason could be fewer convolution layers, which generally better if classes are imbalanced.

It is also noted that EEG signals are known to have a poor signal to noise ratio, and the dataset used in evaluation has been collected in MoBI settings. The MoBI settings create additional challenges in terms of artifacts and even further degradation in the signal-to-noise ratio. Although, the BCINet can perform significantly better compared to the model like EEGNet, DeepConvNet, and ShallowNet in terms of performance and resource requirements. Such a model poses a high potential for BCI applications. These BCI applications do not necessarily need to be limited to a laboratory environment but could be from a real-world environment (MoBI settings).

TABLE III

ACCURACY FOR CC AND PHRC DATASET WITH RESPECT TO EEGNET, ShallowNet, DeEPCONVNET, AND BCINET

$\begin{array}{ccccc}\text { Datasets } & \text { EEGNet } & \text { ShallowNet } & \text { DeepConvNet } & \text { LighConvNet } \\ \text { CC } & 0.6377 & 0.7407 & 0.6881 & 0.7421 \\ \text { pHRC } & 0.7857 & 0.6705 & 0.8973 & 0.9857\end{array}$

\section{LIMITATIONS AND FUTURE WORK}

The BCINet shows several promising properties with high performance compare to the established models. However, to generalize and evaluate the efficacy with all type of BCI applications, several steps are required to take in the future as follows:

1) The current datasets were collected in two distinct MoBI settings but it lacks varieties of other BCI applications; in the future, we will evaluate the efficacy of the model with multiple kinds of BCI datasets such as motor imagery (MI), SSVEP, cognitive workload, etc. with extensive measures [1].

2) In this paper, we have only shown the performance of the BCINet as a whole in terms of performance form the machine learning point of view. In the future work, the output of different layers will be evaluated and compared with traditional EEG features such as topography, eventrelated spectral perturbation (ERSP) [32], etc. as well as evaluation will be performed for each layer such as the impact of hyper-parameters.

3) The current proposed model shows a significantly improved performance with EEG signals. In the future, we also wanted to evaluate this model with similar time-series data such as electrocardiography (ECG), electromyography (EMG), etc. to evaluate efficacy and robustness.

\section{CONCLUSION}

In this paper, we have proposed a novel BCINet model that outperforms the established model like EEGNet, ShallowNet, and DeepConvNet to classify data collected in MoBI setting. We have also shown that BCINet can outperform baseline models with up to $75 \%$ reduction in trainable parameters and up to $20 \%$ improvement in accuracy. Such a model has vast potential in the development of real-world (MoBI) BCI applications with higher performance but with comparatively limited resource requirements.

\section{ACKNOWLEDGMENTS}

This work was supported in part by the Australian Research Council (ARC) under discovery grant DP180100656, and the National Natural Science Foundation of China under Grant 61703399.

\section{REFERENCES}

[1] J. R. Wolpaw, N. Birbaumer, D. J. McFarland, G. Pfurtscheller, and T. M. Vaughan, "Brain-computer interfaces for communication and control," Clinical neurophysiology, vol. 113, no. 6, pp. 767-791, 2002.

[2] M. Tudor, L. Tudor, and K. I. Tudor, "Hans berger (1873-1941)the history of electroencephalography," Acta medica Croatica: casopis Hravatske akademije medicinskih znanosti, vol. 59, no. 4, pp. 307-313, 2005.

[3] B. Burle, L. Spieser, C. Roger, L. Casini, T. Hasbroucq, and F. Vidal, "Spatial and temporal resolutions of eeg: Is it really black and white? a scalp current density view," International Journal of Psychophysiology, vol. 97, no. 3, pp. 210-220, 2015.

[4] A. Craik, Y. He, and J. L. Contreras-Vidal, "Deep learning for electroencephalogram (eeg) classification tasks: a review," Journal of neural engineering, vol. 16, no. 3, p. 031001, 2019.

[5] O. Abdel-Hamid, A.-r. Mohamed, H. Jiang, L. Deng, G. Penn, and D. Yu, "Convolutional neural networks for speech recognition," IEEE/ACM Transactions on audio, speech, and language processing, vol. 22, no. 10, pp. 1533-1545, 2014.

[6] K. He, X. Zhang, S. Ren, and J. Sun, "Deep residual learning for image recognition," in Proceedings of the IEEE conference on computer vision and pattern recognition, 2016, pp. 770-778.

[7] R. Chai, S. H. Ling, P. P. San, G. R. Naik, T. N. Nguyen, Y. Tran, A. Craig, and H. T. Nguyen, "Improving eeg-based driver fatigue classification using sparse-deep belief networks," Frontiers in neuroscience, vol. 11, p. 103, 2017.

[8] D. Lu and J. Triesch, "Residual deep convolutional neural network for eeg signal classification in epilepsy," arXiv preprint arXiv:1903.08100, 2019.

[9] A. Emami, N. Kunii, T. Matsuo, T. Shinozaki, K. Kawai, and H. Takahashi, "Seizure detection by convolutional neural network-based analysis of scalp electroencephalography plot images," NeuroImage: Clinical, vol. 22, p. 101684, 2019.

[10] H. Cecotti and A. Graser, "Convolutional neural networks for p300 detection with application to brain-computer interfaces," IEEE transactions on pattern analysis and machine intelligence, vol. 33, no. 3, pp. 433$445,2010$.

[11] Z. Tayeb, J. Fedjaev, N. Ghaboosi, C. Richter, L. Everding, X. Qu, Y. Wu, G. Cheng, and J. Conradt, "Validating deep neural networks for online decoding of motor imagery movements from eeg signals," Sensors, vol. 19, no. 1, p. 210, 2019.

[12] H. Shan, Y. Liu, and T. P. Stefanov, "A simple convolutional neural network for accurate p300 detection and character spelling in brain computer interface." in IJCAI, 2018, pp. 1604-1610.

[13] F. Lotte, "Signal processing approaches to minimize or suppress calibration time in oscillatory activity-based brain-computer interfaces," Proceedings of the IEEE, vol. 103, no. 6, pp. 871-890, 2015.

[14] S. Hu, Y. Lai, P. A. Valdes-Sosa, M. L. Bringas-Vega, and D. Yao, "How do reference montage and electrodes setup affect the measured scalp eeg potentials?" Journal of neural engineering, vol. 15, no. 2, p. 026013, 2018. 
[15] J. Xue, J. Li, and Y. Gong, "Restructuring of deep neural network acoustic models with singular value decomposition." in Interspeech, 2013, pp. 2365-2369.

[16] C. W. Wu, "Prodsumnet: reducing model parameters in deep neural networks via product-of-sums matrix decompositions," arXiv preprint arXiv:1809.02209, 2018.

[17] H. Pratt, B. Williams, F. Coenen, and Y. Zheng, "Fenn: Fourier convolutional neural networks," in Joint European Conference on Machine Learning and Knowledge Discovery in Databases. Springer, 2017, pp. 786-798.

[18] C. Ding, S. Liao, Y. Wang, Z. Li, N. Liu, Y. Zhuo, C. Wang, X. Qian, Y. Bai, G. Yuan et al., "Circnn: accelerating and compressing deep neural networks using block-circulant weight matrices," in Proceedings of the 50th Annual IEEE/ACM International Symposium on Microarchitecture, 2017, pp. 395-408.

[19] E. Jungnickel and K. Gramann, "Mobile brain/body imaging (mobi) of physical interaction with dynamically moving objects," Frontiers in human neuroscience, vol. 10, p. 306, 2016.

[20] V. J. Lawhern, A. J. Solon, N. R. Waytowich, S. M. Gordon, C. P. Hung, and B. J. Lance, "Eegnet: a compact convolutional neural network for eeg-based brain-computer interfaces," Journal of neural engineering, vol. 15 , no. 5, p. $056013,2018$.

[21] P. Bashivan, I. Rish, M. Yeasin, and N. Codella, "Learning representations from eeg with deep recurrent-convolutional neural networks," arXiv preprint arXiv:1511.06448, 2015.

[22] R. T. Schirrmeister, J. T. Springenberg, L. D. J. Fiederer, M. Glasstetter, K. Eggensperger, M. Tangermann, F. Hutter, W. Burgard, and T. Ball, "Deep learning with convolutional neural networks for eeg decoding and visualization," Human brain mapping, vol. 38, no. 11, pp. 5391-5420, 2017.

[23] A. K. Singh, "Cognitive conflict in virtual reality based object selection task," Ph.D. dissertation, 2019.

[24] A. K. Singh, K. Gramann, H.-T. Chen, and C.-T. Lin, "The impact of hand movement velocity on cognitive conflict processing in a $3 \mathrm{~d}$ object selection task," BioRxiv, 2020.

[25] S. Aldini, A. Akella, A. K. Singh, Y.-K. Wang, M. Carmichael, D. Liu, and C.-T. Lin, "Effect of mechanical resistance on cognitive conflict in physical human-robot collaboration," in 2019 international conference on robotics and automation (ICRA). IEEE, 2019, pp. 6137-6143.

[26] J. E. Trost, "Statistically nonrepresentative stratified sampling: A sampling technique for qualitative studies," Qualitative sociology, vol. 9 , no. 1 , pp. 54-57, 1986.

[27] D. P. Kingma and J. Ba, "Adam: A method for stochastic optimization," arXiv preprint arXiv:1412.6980, 2014.

[28] M. Abadi, P. Barham, J. Chen, Z. Chen, A. Davis, J. Dean, M. Devin, S. Ghemawat, G. Irving, M. Isard et al., "Tensorflow: A system for largescale machine learning," in 12th $\{$ USENIX $\}$ symposium on operating systems design and implementation (\{OSDI\} 16), 2016, pp. 265-283.

[29] A. Gulli and S. Pal, Deep learning with Keras. Packt Publishing Ltd, 2017.

[30] Z. Qin, Z. Zhang, D. Li, Y. Zhang, and Y. Peng, "Diagonalwise refactorization: An efficient training method for depthwise convolutions," in 2018 International Joint Conference on Neural Networks (IJCNN). IEEE, 2018, pp. 1-8.

[31] D. J. McFarland, W. A. Sarnacki, and J. R. Wolpaw, "Brain-computer interface (bci) operation: optimizing information transfer rates," Biological psychology, vol. 63, no. 3, pp. 237-251, 2003.

[32] S. Makeig and T.-P. Jung, "Tonic, phasic, and transient eeg correlates of auditory awareness in drowsiness," cognitive brain research, vol. 4, no. 1, pp. 15-25, 1996. 